\title{
Laser Pulse Compression Towards Collapse and Beyond in Plasma
}

T C Wilson ${ }^{1}, \mathbf{F}$ Y Li ${ }^{1}, \mathbf{S}$ M Weng $^{2,3}, \mathbf{M} \mathbf{C h e n}^{2,3}, \mathbf{P}$ McKenna $^{1}$, Z M Sheng ${ }^{1,2,3}$

${ }^{1}$ SUPA, Department of Physics, University of Strathclyde, Glasgow G4 0NG, United Kingdom

${ }^{2}$ Key Laboratory for Laser Plasmas (MoE) and School of Physics and Astronomy, Shanghai Jiao Tong University, Shanghai 200240, China

${ }^{3}$ Collaborative Innovation Center of IFSA, Shanghai Jiao Tong University, Shanghai 200240, China

\section{Submitted to: J. Phys. B: At. Mol. Phys.}

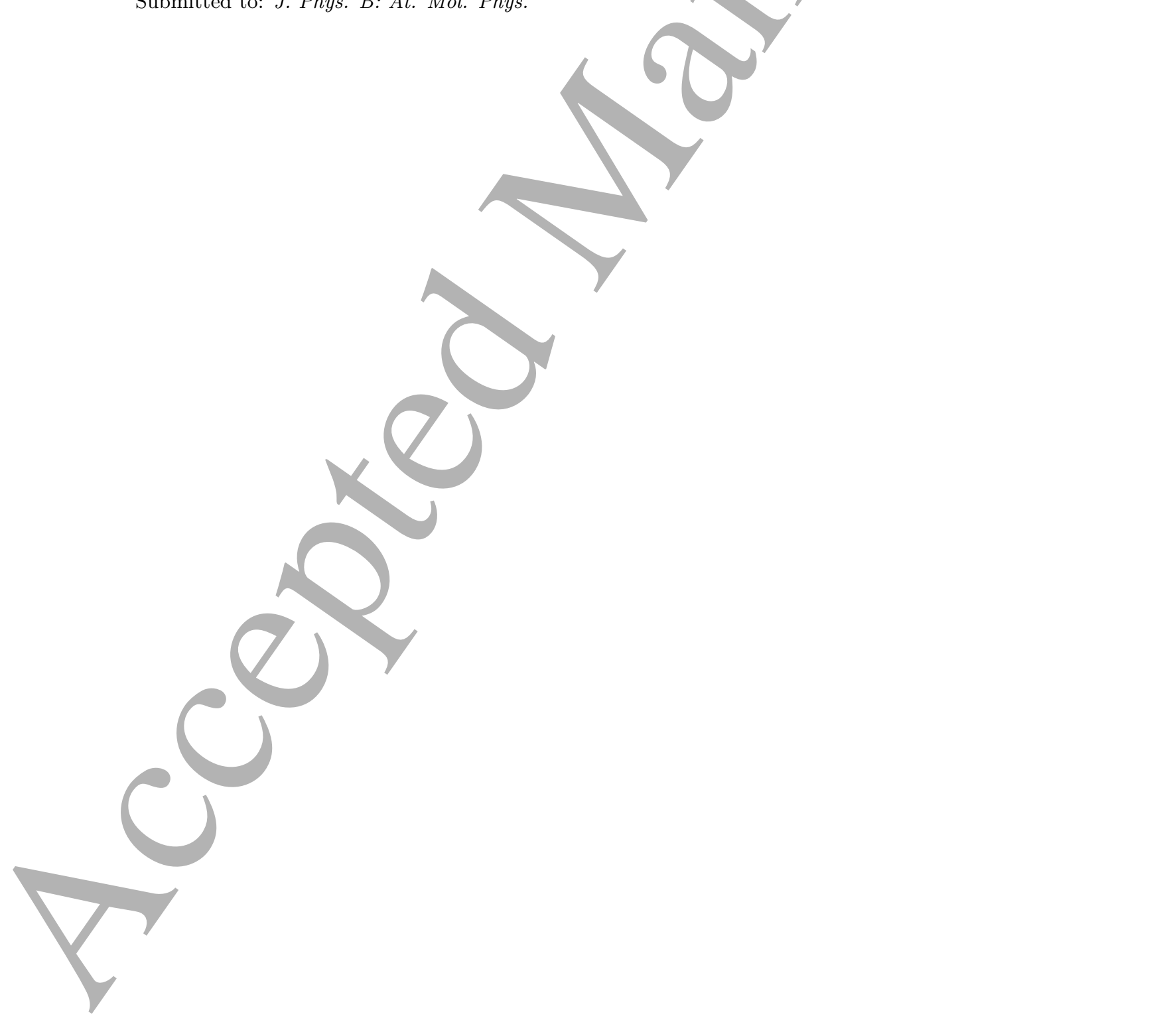


Abstract. The dynamics of three-dimensional (3D) compression of ultrashort intense laser pulses in plasma is investigated theoretically and numerically. Starting from the slowly-varying envelope model, we derive equations describing the spatiotemporal evolution of a short laser pulse towards the singularity, or collapse, based on the variational method. In particular, the laser and plasma conditions leading to spherical compression are obtained. 3D particle-in-cell simulations are carried out to verify these conditions, which also enable one to examine the physical processes both towards and beyond the pulse collapse. Simulations suggest that the laser pulse can be spherically compressed down to a minimum size of the order of the laser wavelength, the so called lambda-cubic regime. The compression process develops over twice as fast in simulation than is predicted by the envelope model, due to the simplified nature of the latter. The final result of this process is pulse collapse, which is accompanied with strong plasma density modulation and spectrum broadening. The collapse can occur multiple times during the laser pulse propagation, until a significant part of the pulse energy is dissipated to electron acceleration by the laser ponderomitve force. It is also shown that a strong external DC magnetic field applied along the laser propagation direction can enhance the rate of compression for circularly-polarised laser pulses, when compared to an unmagnetised plasma, allowing access to strong compression and focusing in the low-density and low-amplitude regime.

\section{Introduction}

The ultrashort laser pulse has become a routine tool of high energy-density physics research, but the nature of bandwidth-limited amplification, and the finite damage threshold of solid-state optics, place lower limits on the pulse duration, focal spot size, and thus an upper limit on the peak intensity which can be produced. Plasmas are a natural choice to move beyond these limits, having extremely tunable optical properties and almost no restrictions on the beam intensities which may be sustained within them.

The study of nonlinear optical materials, as a medium to focus and compress already ultrashort laser pulses, has been a subject of considerable research interest in the past decades $[1,2,3,4,5,6,7,8,9,10,11,12]$. Generally, this can be described by the nonlinear Schrödinger equation for the envelope of the laser pulse under the paraxial approximation $[1,2,3,13]$. This equation predicts that simultaneous selffocusing and compression can lead to collapse of laser pulses, where the laser focal spot size and duration both approach zero, accompanied by explosive growth in the laser intensity. Physically, however, such a limit cannot be achieved since the envelope equation is no longer valid when the laser pulse is approaching collapse. A modified nonlinear Schrödinger equation demonstrates the phenomenon of pulse steepening and spectrum broadening in various optical media $[14,15]$.

Compared to other nonlinear optical media, plasma can be used to manipulate short laser pulses at high peak power well above tens of terawatt. Under such peak powers, the nonlinear effects are mainly due to the relativistic effect and ponderomotive force. In this case, the envelope evolution of the laser pulses can still be described by a modified nonlinear Schrödinger equation. Typically this equation can be studied analytically $[16,17,6,7]$ or numerically $[18,4]$. Through these combined efforts, relativistic self-focusing and self-compression are well understood. However, the two processes have mostly been considered separately [19]. For ultrashort pulses, these processes often occur simultaneously and therefore, in order to accurately reproduce the problem a coupled treatment is required. An interesting idea is to focus

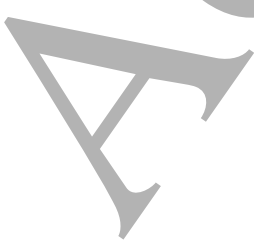




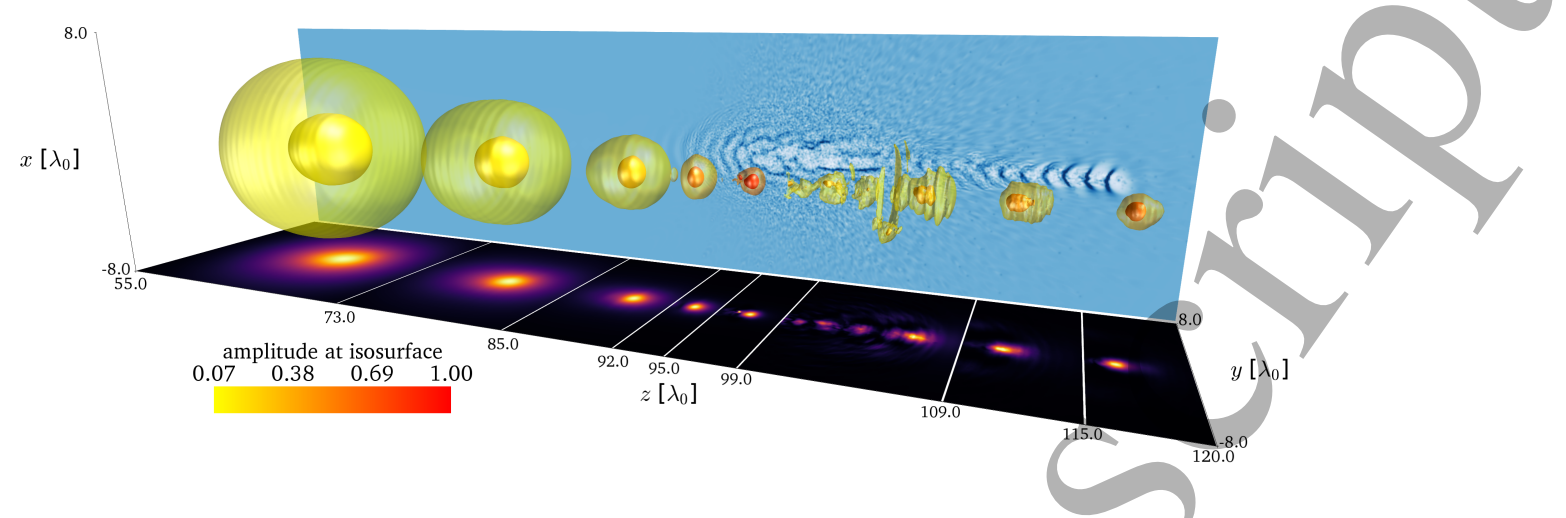

Figure 1: Three-dimensional spherical compression of an ultrashort pulse towards collapse and beyond, shown at progressive times, for the parameter setup discussed in section 3.1. The isosurfaces correspond to $1 / \mathrm{e}$ (transparent) and $2 / \mathrm{e}$ (solid) times the peak amplitude of the snapshot, coloured according to the lower-left colourbar. The bottom projection shows intensity slices in the $y-z$ plane at $x=0$, with different snapshots separated by white lines. The rear plane shows the electron density distribution in the $x-z$ plane at $y=0$, for the $t=192 \tau_{0}$ snapshot. We see dramatic compression of the initial pulse towards the lambda-cubic regime at $z \sim 100 \lambda_{0}$, accompanied by a very large increase in intensity, over 150 times the initial value. Further attempts to compress the pulse lead to pulse collapse, distortion and strong ponderomotively-driven electron caviation. Despite this, the pulse remnant re-emerges from the collapse site, continues propagating, and undérgoes further collapses (here shown up to the second, at $z 120 \lambda_{0}$ ) until the pulse energy is significantly depleted. The rendered snapshots are captured at $t=120,140,153,159,164,177,184$ and $192 \tau_{0}$ respectively, shown in order from left to right.

and compress laser pulses to the so called lambda-cubic regime [20], which may provide the opportunity to produce isolated attosecond pulses and achieve extreme high intensity $[21,22,23]$. When approaching this regime, the paraxial envelope equation is no longer valid. More interestingly, laser pulse collapse may develop during its focusing and compression, which is expected to be accompanied by spectrum broadening, plasma heating or particle acceleration and plasma density modulation. These are notoriously difficult to account for in the envelope model. Self-consistent numerical simulation using the particle-in-cell (PIC) method, is an effective way to investigate this problem in 3D geometry. PIC simulation allows one to resolve subwavelength structures of the laser pulse during the collapse, which is critical to understanding this process. A typical picture of this, leading up to and beyond collapse, is shown in figure 1.

In this work we investigate the process of laser pulse compression in 3D towards the pulse collapse both analytically and numerically. We start with the paraxial model of envelope evolution in $3+1$ (three spatial plus one temporal) dimensions. By applying the variational approach to the slowly-varying envelope equation, the conditions are derived both in unmagnetised and magnetised plasmas, under which the rates of transverse and longitudinal evolution is matched and the pulse evolves spherically towards the lambda-cubic regime and finally towards collapse. This model provides guidance for self-consistent 3D PIC simulations, which can reveal various

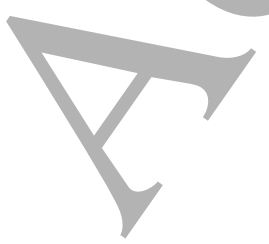


features during the laser pulse evolution towards collapse and beyond.

\section{Theory Model for Laser Pulse Compression in 3D}

We consider a right-circularly polarised (R-mode) laser pulse, and are interested in the evolution of the pulse envelope $\psi$ in time and space, so we adopt the routinely used slowly-varying envelope approximation $[4,6,19]$ with the following nonlinear Schrödinger equation

$$
2 \mathrm{i} \frac{\partial \psi}{\partial z}+\nabla_{\perp}^{2} \psi+\epsilon_{1} \frac{\partial^{2} \psi}{\partial \tau^{2}}+\epsilon_{2}|\psi|^{2} \psi=0
$$

where $\nabla_{\perp}^{2}$ is the tranverse Laplacian and $\epsilon_{1}$ and $\epsilon_{2}$ are constants. We have transformed to comoving time $\tau=t-z / v_{g}$ and are working with the dimensionless variables $\tilde{r}=r k_{p}, \tilde{z}=z k_{p}^{2} / k$ and $\tilde{\tau}=\tau \omega_{p}$, where $k$ and $k_{p}=\omega_{p} / c$ are the laser and plasma wavenumbers respectively, $v_{g}$ is the laser group velocity and the tildes are henceforth dropped for convenience. Note that the constants $\epsilon_{1}=\beta_{g}^{-2}-1$ and $\epsilon_{2}=\left(1-\omega_{c} / \omega_{0}\right)^{-4} / 2$, where $\beta_{g}=v_{g} / c$ is the normalised laser group velocity, $\omega_{c}=e B / m_{e}$ is the plasma electron cyclotron frequency in the case when an external DC magnetic field with strength $B$ is applied along the laser propagation direction and $\omega_{0}$ is the central laser frequency. The generalised group velocity for R-mode laser light may be derived from the dispersion relation [19] and is found to be

$$
\beta_{g}=\left[1-\frac{\omega_{p}^{2}}{\omega_{0}^{2}-\omega_{0} \omega_{c}}\right]^{1 / 2}\left[1+\frac{\omega_{c} \omega_{p}^{2}}{2 \omega_{0}\left(\omega_{0}-\omega_{c}\right)^{2}}\right]^{-1},
$$

which reduces to the usual plasma group velocity when $\omega_{c}=0$.

We next apply the variational method [16] to study the evolution of $\psi$. We choose a Gaussian ansatz for $\psi$ of the form

$$
\psi(r, z, \tau)=a \exp \left[-\frac{r^{2}}{R^{2}}-\frac{\tau^{2}}{D^{2}}+\mathrm{i} \rho r^{2}+\mathrm{i} \phi \tau^{2}\right],
$$

where $R, D, \rho$ and $\phi$ describe the beam spot size, duration, radial curvature and longitudinal chirp respectively and are all real functions of $z$. Here $a$ is the normalized peak amplitude and is a complex function of $z$, which is related to the laser intensity $I$ $\left(\mathrm{W} / \mathrm{cm}^{2}\right)$ and wavelength $\lambda_{0}(\mu \mathrm{m})$ via $I \lambda_{0}^{2}=|a|^{2}\left(2.76 \times 10^{18} \mathrm{~W} / \mathrm{cm}^{2} \mu \mathrm{m}^{2}\right)$. Assuming evolution from initial conditions $a_{0}, R_{0}$ and $D_{0}$, where $R_{0}$ is normalised to $k_{p}$ and $D_{0}$ is normalised to $\omega_{p}$. It is convenient to further normalise all parameter functions to their initial values, e.g. $\tilde{R}=R / R_{0}, \tilde{D}=D / D_{0}, \tilde{a}=a / a_{0}$, with the tildes dropped henceforth for convenience. This treatment eventually yields two coupled equations describing the transverse and longitudinal evolution of $\psi$, given by

$$
\begin{aligned}
& \frac{\mathrm{d}^{2} R}{\mathrm{~d} z^{2}}=\frac{4}{R_{0}^{4} R^{3}}\left[1-\frac{\mathcal{E}_{0} \epsilon_{2}}{8 \sqrt{2} D_{0} D}\right], \\
& \frac{\mathrm{d}^{2} D}{\mathrm{~d} z^{2}}=\frac{4 \epsilon_{1}^{2}}{D_{0}^{4} D^{3}}\left[1-\frac{\mathcal{E}_{0} \epsilon_{2} D_{0} D}{8 \sqrt{2} \epsilon_{1} R_{0}^{2} R^{2}}\right],
\end{aligned}
$$

with the constant of motion, i.e., energy conservation given by $\mathcal{E}_{0}=\left|a_{0}\right|^{2} R_{0}^{2} D_{0}$. Equations (3) and (4) describe the evolution of the pulse spot size and duration respectively, and are coupled to each other inextricably. In limiting cases we may 

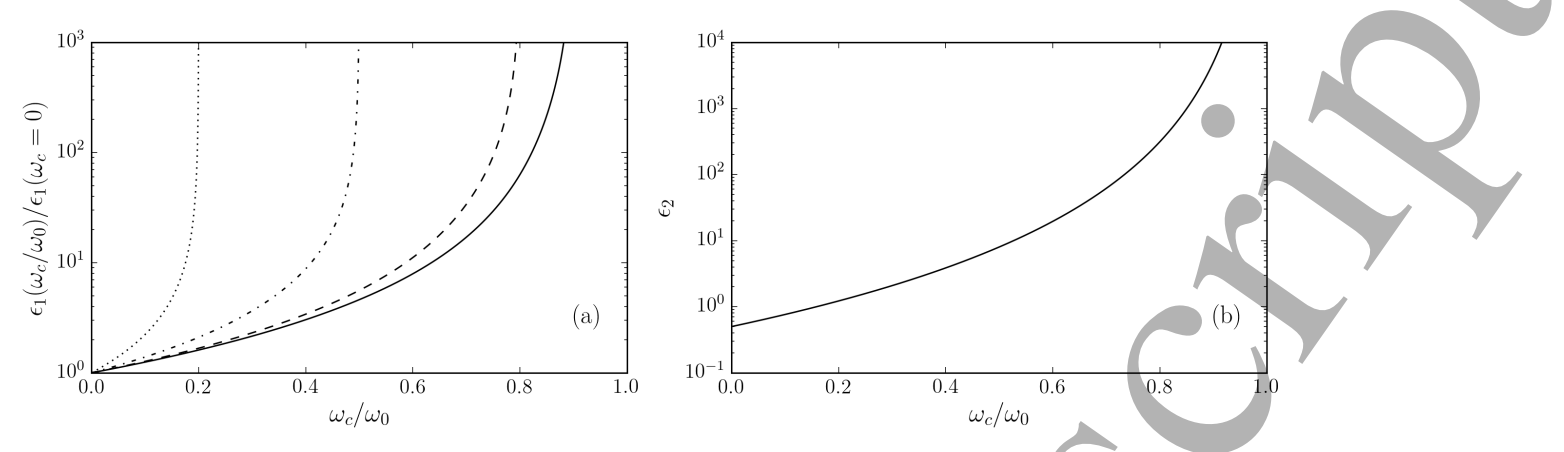

Figure 2: Scaling of the two NLSE coefficients with external magnetic field strength. (a) The scaling of $\epsilon_{1}$ with magnetic field strength as compared to the case of no magnetic field. The scaling is stronger at higher plasma density. The solid, dashed, dot-dashed and dotted lines correspond to $n_{e} / n_{c}=0.1,0.2,0.5$ and 0.8 respectively. (b) The scaling of $\epsilon_{2}$ with magnetic field strength, tending to infinity as $\omega_{c} / \omega_{0} \rightarrow 1$.

simplify this problem. For instance, if we consider the simple case of no external magnetic field, then $\epsilon_{1}$ depends only on the plasma density and $\epsilon_{2}=0.5$. Under these circumstances we can see immediately that/self-compression of the laser is difficult when the plasma is underdense, as $\epsilon_{1}^{2} \ll 1$. This is compounded upon when $D_{0} \gg 1$, we see that $\mathrm{d}^{2} D / \mathrm{d} z^{2} \approx 0$, and may consider changes to $R$ only, resulting in the familiar $2 \mathrm{D}$ self-focusing regime. Conversely, if $R_{0} \gg D_{0} \gg 1$ and the plasma is of sufficient density that $\epsilon_{1}^{2} \sim 1$ then self-compression dominates, and we may consider $R$ to be fixed instead $[24,25]$.

The picture is more complicated when there is an external magnetic field. Both $\epsilon_{1}$ and $\epsilon_{2}$ scale with the magnetic field strength, shown in Figure 2. We can therefore see that while $\epsilon_{1}^{2}$ remains small for unmagnetised low-density plasma, this is not the case when a strong magnetic field is introduced. In the same vein, $\epsilon_{2}$ scales very strongly with magnetic field strength. As such, we now have a system in which is more sensetive to relativistic nonlinearlty through $\epsilon_{2}$, and more suceptable to self-compression specifically through $\epsilon_{1}$. This combination results in the onset of relativistic effects at lower laser intensity (when compared to the unmagnetised case), and allows for self-compression in low-density regimes in which it is typically prohibited.

In such a sensitive regime, the coupling between (3) and (4) becomes important. For instance, while a pulse may begin at an amplitude at which self-compression is negligible, the effect of self-focusing may push the amplitude up to the point at which appreciable compression begins to occur, and this in turn will further influence the self-focusing.

A special case of this problem is found when considering spherically symmetric evolution, i.e. when $R(z) \equiv D(z)$. This has been studied previously for spherically symmetric geometry, considering only a single radial dimension [26]. We point out that the result obtained in spherical coordinates is reproducible in cartesian coordinates by applying the initial condition

$$
D_{0}^{2}=\epsilon_{1} R_{0}^{2}
$$

A subtlety of this condition is that it fixes the relative shape of the pulse for 
the entire evolution, with the curves $R(z)$ and $D(z)$ only intersecting at the point of singularity. For any pulse aspect ratio other than unity, this implies $R(z)$ and $D(z)$ evolve asymmetrically. While it is convenient to call this the condition for spherical compression, it is not neccesarily spherical in real units. This allows for a situation where, for example, $D$ may reduce to a single wavelength, but $R$ may still be several times larger if the aspect ratio is low.

If reaching the lambda-cubic regime is our goal, it is tempting to suggest we should tune the pulse so that $R(z)$ and $D(z)$ intersect at $\lambda_{0}$. This tuning may be done with ease by inspection of the solutions to (3) and (4). The result is a small change to either $R_{0}$ or $D_{0}$ to nudge the point of intersection above zero. In fact, the difference between a pulse satisfying this condition and one satisfying the 'spherical' condition is incredibly small, and the envelope model is simply not powerful enough for such a fine detail to carry over into simulations (let alone experiments) and not be consumed by more dominant competing processes and noise. As such, taking (5) as the condition for spherical compression is justified.

To apply this model to more realistic situations, one must remember that the values for $D_{0}$ are assumed to be in-plasma, therefore the change in duration due to group velocity reduction should be taken into account as a real pulse enters a plasma. As this happens, a pulse with vacuum duration $D_{0}^{\mathrm{vac}}$ will be shortened to $D_{0}=\beta_{g} D_{0}^{\mathrm{vac}}$. Now, the adjusted rate-matching conditions are given by

$$
D_{0}^{\mathrm{vac}}=R_{0}\left(\beta_{g}^{-4}-\beta_{g}^{-2}\right)^{1 / 2}
$$

Allowing both $\omega_{p}$ and $\omega_{c}$ to vary in (6) via the group velocity $\beta_{g}$, we can show how the rate-matching condition changes with both density/and magnetic field strength, as illustrated in figure 3. In general, lower plasma densities and lower magnetic fields require shorter pulses or broader laser spots in order to compress spherically. Specifically, in an unmagetised underdense plasma with $\omega_{p}^{2} \ll \omega_{0}^{2}$, this requires $D_{0}^{\text {vac }} / R_{0} \ll 1$, i.e., the initial pulse length must be much shorter than the laser transverse spot size. Only when the plasma is of near-critical density, one can realise spherical compression with initial $D_{0}^{\mathrm{vac}} / R_{0} \geq 1$. On the other hand, when a sufficiently high external DC magnetic field is applied, one may still achieve spherical compression with initial $D_{0}^{\text {vac }} / R_{0} \sim 1$, even if the plasma is quite underdense. This implication is applicable to R-mode laser light exclusively. Left-circularly polarised, or linearly polarised light behaves entirely differently when an external magnetic field is imposed. L-mode laser light sees the opposite effect as R-mode, where the required aspect ratio becomes even smaller, and linearly polarised light experiences much more complicated effects, behaving as a superposition of $\mathrm{R}$ and $\mathrm{L}$ mode light [27]. Equation (5) describes the conditions for spherical compression for L-mode and linearly polarised light only when $\omega_{c}=0$.

Initial beam tendency may be predicted from (3) and (4) as critical powers, taking $R(0)=1$ and $D(0)=1$,

$$
\begin{aligned}
& P_{R}=2.463 \times 10^{10}\left(1-\frac{\omega_{c}}{\omega_{0}}\right)^{4} \frac{\omega_{0}^{2}}{\omega_{p}^{2}} \mathrm{~W} \\
& P_{D}=2.463 \times 10^{10}\left(1-\frac{\omega_{c}}{\omega_{0}}\right)^{4} \frac{\omega_{0}^{2}}{\omega_{p}^{2}} \frac{\epsilon_{1} R_{0}^{2}}{c^{2} D_{0}^{2}} \mathrm{~W},
\end{aligned}
$$

with $R_{0}$ and $D_{0}$ now in real units. The values of $P_{R}$ and $P_{D}$ are derived using the approximate beam power for a CP Gaussian laser $P=4.298 \times 10^{10}\left|a_{0}\right|^{2} R_{0}^{2} / \lambda_{0}^{2} \mathrm{~W}$, 

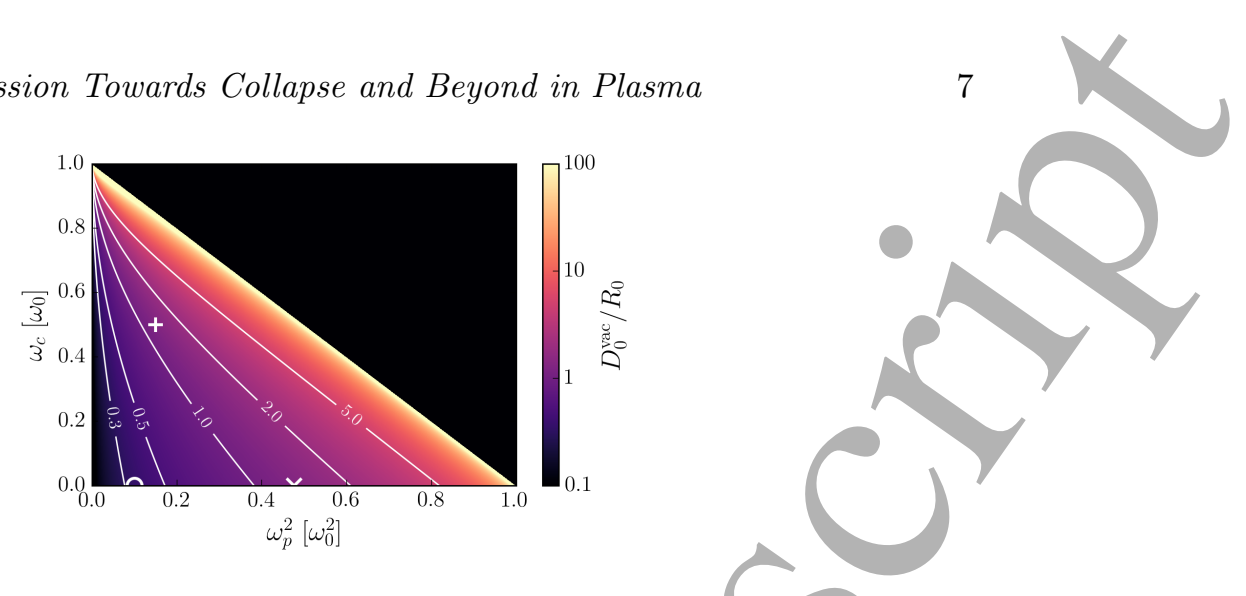

Figure 3: Initial pulse aspect ratio required for spherical compression as a function of density and cyclotron frequency. The isobars show the curves for some specific ratios. The upper right black area of the plot is beyond the effective critical density, and hence returns non-real values. The cross, plus, and circle signs denote the conditions we choose to simulate.

where both $\lambda_{0}$ and $R_{0}$ are again in real units. Physically, Equations (7) and (8) give the power thresholds above which the laser pulse will immediately begin to transversely focus or longitudinally compress respectively, These two thresholds are often different, and the initial tendency will not neccesarily be maintained. A laser pulse with power satisfying $P_{R}<P<P_{D}$ will initially focus transversely and spread longitudinally, but recall the coupled nature of (3) and (4); over the course of focusing the peak amplitude may rise enough that it begins to compress, reversing this initial tendency. If (5) is satisfied, the two thresholds become identical, and if this threshold is exceeded the pulse will collapse towards the lambda-cubic regime.

We note that in (7) and (8), the constant value is a factor of $\sqrt{2}$ higher than the usually cited threshold power for self-focusing [5]. This is due to the variational method itself. The dimensionality of the problem, and the choice of ansatz used, introduce a constant factor to the second term of the RHS of (3) and (4). For a Gaussian ansatz and 3 spatial dimensions this factor is equal to $(3 \sqrt{2})^{-1}$. A different ansatz, or a different number of spatial dimensions will change this value. This inconsistency is well documented in reference [26], comparing both Gaussian and sech profiles in up to three spatial dimensions, and is a result of the assumption that the laser profile remains abberationless. This artefact of approximation acts to inflate the calculated power thresholds and stretch the numerical solutions of (3) and (4) for higher-dimensional systems.

\section{Results from PIC simulation}

Even though (6) gives the conditions for 3D compression in plasma, it does not provide details on how it develops. In order to test these equations and show the physical processes involved, simulations are performed using both the 3D PIC code OSIRIS [28] and supplemented by the cylindrically symmetric, quasi-3D PIC code FBPIC [29], which can produce very similar results to that of cartesian 3D PIC codes for the problems considered here, while at the same time being computationally cost-effective. A more detailed comparison between the two codes is given in the supplementary material, where we find good agreement between the two for the purposes of this 
work.

In general, length scales are normalised to the laser wavelength in vacuum $\lambda_{0}$, and time scales to the laser period $\tau_{0}=\lambda_{0} / c$. We note that in the following simulation results, 'time' now refers to elapsed simulation time, rather than the comoving time used in section 2. In OSIRIS, a spatial resolution of $16 \times 16 \times 16$ cells $/ \lambda_{0}^{3}$ is used to ensure the subwavelength structures can be clearly resolved. The simulation includes electrons and ions, with $m_{i} / m_{e}=1836$ representing a hydrogen plasma. Each cell contains 1 electron and 1 ion, with quartic particle shapes. The laser is right-circularly polarised, initialised from the wall in vacuum a distance of $5 \lambda_{0}$ from the start of the plasma, then traverses a short linear density ramp of length $1 \lambda_{0}$ before entering a cold homogeneous plasma with the plasm plateau beginning at $z=0$. The simulation box is $200 \lambda_{0}$ in total along $z, 50 \lambda_{0} \times 50 \lambda_{0}$ in transverse extent. In FBPIC, we maintain the resolution of $16 \times 16$ cells $/ \lambda_{0}^{2}$ in the $z-r$ plane and simulate two azimuthal modes. We again simulate both electrons and ions use a total of 40 particles per species per cell, distributed as $2 \times 2 \times 10$ per-cell along $z, r$ and $\theta$ using cubic particle shapes. Laser amplitude, static magnetic field strength, and plasma density is varied between simulations.

\subsection{The case for unmagnetised plasma}

First, we simulate a non-magnetised plasma, with a plateau density of $n_{0}=\omega_{p}^{2} / \omega_{0}^{2}=$ 0.475 and an initial amplitude of $a_{0}=0.12$, corresponding to $P / P_{c} \approx 1.2$. The laser focal plane is set to the start of the plasma density plateau, with the beam waist $R_{0}=10 \lambda_{0}$, and vacuum duration $D_{0}^{\text {vac }}=13 \tau_{0}$. These conditions are tailored to satisfy the matching condition $D_{0}^{\mathrm{vac}} / R_{0}=1.3$ as denoted by the cross sign on figure 3 . Figure 4 shows the overview of the pulse duration, amplitude evolution, final electron density and frequency spectrum over the course of the simulation. Firstly, the theory predicts collapse down to a singularity, and this is borne out in the simulation. Shown in figure 4(a), the functions $R$ and $D$ evolve at close-to the same rate, which is in good agreement with the theory. However, the overall rate at which the pulse collapses is much faster than predicted by the envelope model given in (3) and (4), implying both (7) and (8) are overestimated. We attribute this to artificial threshold inflation due to the 3D nature of the problem, discussed at the end of section 2. Accompanying the 3D compression in the simulation, the peak intensity is enhanced by two orders of magnitude. Despite this being a significant increase, it falls short of what a simple analysis might suggest.

If we say that the pulses are compressed to the lambda-cubic (i.e. $R=D=1$ ), and there is no energy loss, then the maximum amplification factor we might see is a function only of the initial pulse dimensions, $a / a_{0}=\sqrt{R_{0}^{2} D_{0}}$. This results in a predicted amplification factor of 30 times the amplitude, or 900 times the intensity for the pulse we simulate here. These theoretical predictions are of course, not realistic, as they assume no loss to the plasma, and a perfectly abberationless collapse. Figure 6 shows the energy balance over time of an FBPIC simulation with equivalent parameters. We see that after the pulse has entered the plasma, the energy is partitioned between the laser and particles as expected, with no further loss up the the point of first collapse at around $t=160 \tau_{0}$. Despite this, the amplification falls well short of the (naïvely) predicted result. This is due to both the partitioning of energy between the laser and plasma, and the pulse profile deviating from its initial radial profile, instead arriving at a characteristic shape in which significantly more

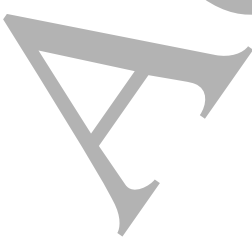



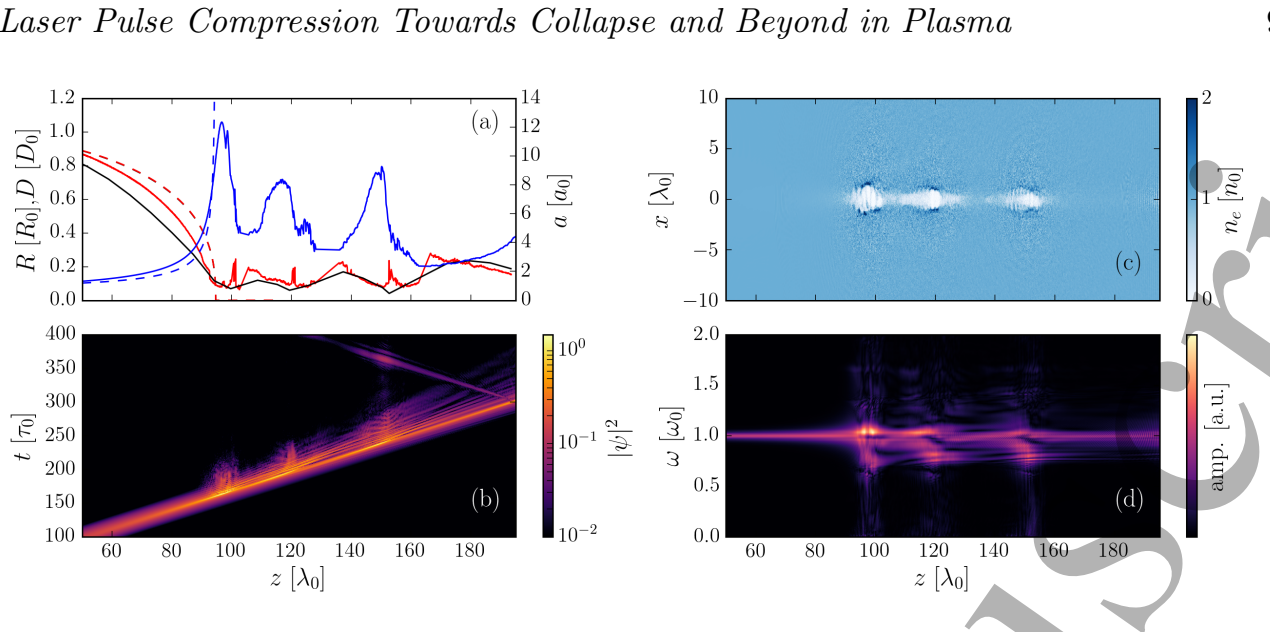

Figure 4: Unmagnetised simulation results. (a) Envelope parameter function evolution over the course of the simulation. The relative changes to $R$ (black), $D$ (red) and $a$ (blue) are shown, the dashed lines show the theory-predicted evolution for the same parameters, in which the $z$ axis has been scaled down by a factor of 3.5 in order to overlay with the simulation results. Immediately following a collapse the pulse becomes highly distorted and so meaningful evaluations of its width and duration are difficult to make in these regions. (b) The laser envelope profile along the central $z$ axis at progressive times. Note that a logarithmic scale has been adopted to depict the broad range of intensity variation through the course of laser pulse collapse, and the reversed light tracks in the top-right are caused by boundary reflections as the pulse leaves the simulation box at around $t=300 \tau_{0}$. (c) Electron density plots at the end of the simulation $\left(t=400 \tau_{0}\right)$. (d) Frequency spectra of the on-axis $B_{x}$ fields performed at fixed longitudinal positions, over the whole simulation time domain $\left(400 \tau_{0}\right)$.

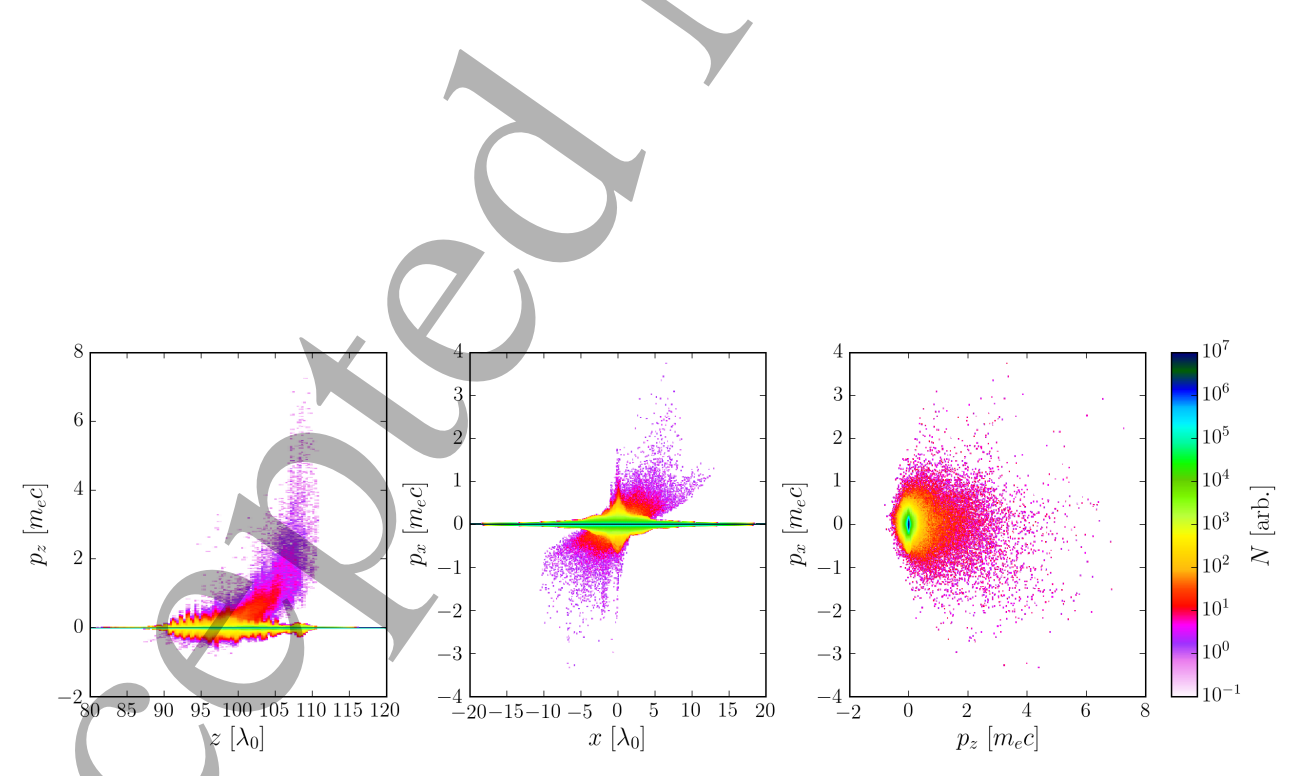

Figure 5: Unmagnetised simulation electron phase-space distributions at $t=180 \tau_{0}$, following the first collapse. (a) The longitudinal momentum $p_{z}$ versus the longitudinal axis $z$. (b) The transverse momentum $p_{x}$ versus the $x$ axis. (c) $p_{z}-p_{x}$ distribution. 


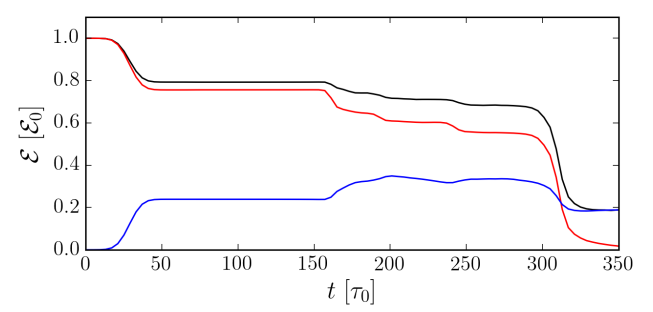

Figure 6: Energy balance over the course of the simulation with $n_{0}=0.475, B=0$. The black line shows the total EM energy contained in the simulation $\left(E^{2}+B^{2}\right)$, the red line shows the total radiative energy calculated from the Poynting vector $(|\boldsymbol{E} \times \boldsymbol{B}|)$, and the blue line shows the total kinetic energy of the electrons (ion kinetic energy is negligible). The sharp drop in EM energy at $t \approx 310 \tau_{0}$ corresponds to the laser pulse leaving the simulation box.

energy is distributed in the wings of the pulse than a Gaussian shape known as the Townes mode $[4,30]$, thus further reducing the potential amplification.

Secondly, successive collapses are found after the first collapse, as the pulse undergoes alternating strong focusing (and compression) and strong diffraction (and decompression), losing energy each time, as seen in figure 6 at $t>160 \tau_{0}$. It is reasonable to extrapolate that this process will continue until there is no longer sufficient energy left in the core of the pulse remnant, at which point it will diffract away rapidly.

Thirdly, the pulse is not reaching a singularity, but a minimum spatial extent of approximately $1 \lambda_{0}^{3}$. This is due to electron cavitation which acts to prevent further compression. The pulse undergoes collapse three times over the course of the simulation, each time accompanied by a burst of $\mathrm{keV}$ to $\mathrm{MeV}$ electrons expelled from the cavity in the forward direction, seen in figure 5(a). There is a high divergence associated with the burst as illustrated by figure $5(\mathrm{~b}-\mathrm{c})$ and the particles spread out in a cone from the point of collapse. Ion motion on the other hand is sedentary, with no suprathermal ions produced over the course of the simulation. At the end of the simulation, the ion density distribution is very close to that of the electrons.

The rapid nature of the cavitation causes a density pileup to form in front of the pulse in the few periods leading up to collapse. This scatters the wave, resulting in a sudden, strong frequency downshift [31, 32], as seen in figure 4(d). In addition to frequency downshift, frequency upshift is also found, which is most obvious near the first collapse. This much broadened spectrum also suggests the occurrence of laser pulse compression down to the lambda-cubic regime.

The ponderomotive force becomes quite strong in the lead-up to collapse, causing the formation of an electron density cavity. The downshifted wave becomes trapped in this newly-formed cavity as a soliton, which decays in turn to a post-soliton. Postsoliton formation in plasmas have been examined in both 2D [33,34], and 3D geometry [35] under a variety of conditions. We observe behaviour consistent with previous studies of 3D post-solitons. Formation of the ion density cavity transfers energy from the trapped EM wave to the particles, resulting in rapid decay of the post-soliton over a few tens of laser periods. Ion motion is also critical to the cavity stability. Acting in reaction to the space charge separation, ions are gradually expelled outwards and, as 

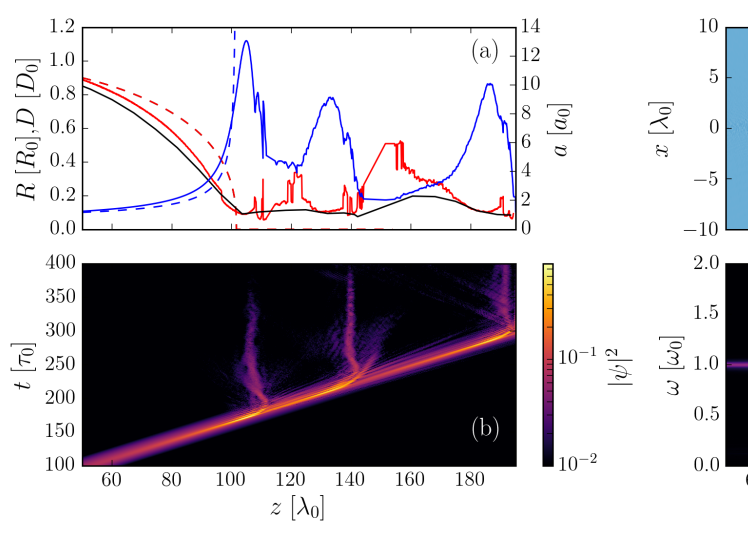

Figure 7: Magnetised simulation results. (a) Envelope parameter function evolution over the course of the simulation. The relative changes to $R$ (black), $D$ (red) and $a$ (blue) are shown, the dashed lines show the theory-predicted evolution for the same parameters, in which the $z$ axis has been scaled down by a factor of 2.6 in order to overlay with the simulation results. Immediately following a collapse the pulse becomes highly distorted and so meaningful evaluations of its width and duration are difficult to make in these regions. (b) The laser envelope profile along the central $z$ axis at progressive times. Note that a logarithmic scale has been adopted to depict the broad range of intensity variation through the course of laser pulse collapse. (c) Electron density plots at the end of the simulation $\left(t=400 \tau_{0}\right)$. (d) Frequency spectra of the on-axis $B_{x}$ fields performed at fixed longitudinal positions, over the whole simulation time domain $\left(400 \tau_{0}\right)$.

such, the cavities continue to slowly expand long after the postsoliton has completely decayed. Figure 4(c) shows the density snapshot at the end of the simulation where the three cavities structures remain well intact.

\subsection{The case for magnetised plasma}

Next, we consider a magnetised casse, with normalised magnetic field strength $\omega_{c} / \omega_{0}=$ 0.5 , plasma density $n_{0}=0.15$ and initial amplitude $a_{0}=0.06$, giving $P / P_{c} \approx 1.5$. We note that without a magnetic field, this plasma and laser amplitude would have a power ratios of $P / P_{R} \approx 0.09$ and $P / P_{D} \approx 0.48$. Even accounting for the inflated thresholds, we would expect this pulse to monotonically diffract and spread in an unmagnetised plasma. This case is denoted by the plus sign on figure 3 and again calls for an aspect ratio of $1.3 \mathrm{in}$ order to have spherical compression. Results in line with those given for the previous simulation are shown in figures 7 and 8 . Similar to the case of unmagnetised plasma, the transverse and longitudinal sizes of the laser pulse reduce at the same rate leading to symmetrical collapse as shown in figure 7(a), which agrees with our theory. Meanwhile, the pulse compression again develops much faster than that predicted by the theory model given in (3) and (4). Even with such a low laser energy and in low-density plasma conditions that would typically by unfavourable for eompression, pulse evolution towards collapse is found. Again, the pulse undergoes collapse several times, as seen in figure $7(\mathrm{~b})$, each time losing small amounts of energy to energetic particle production as seen in figure 9. This case also exhibits post-

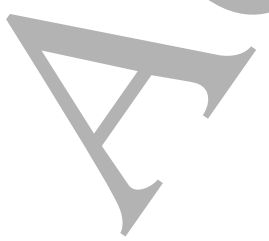



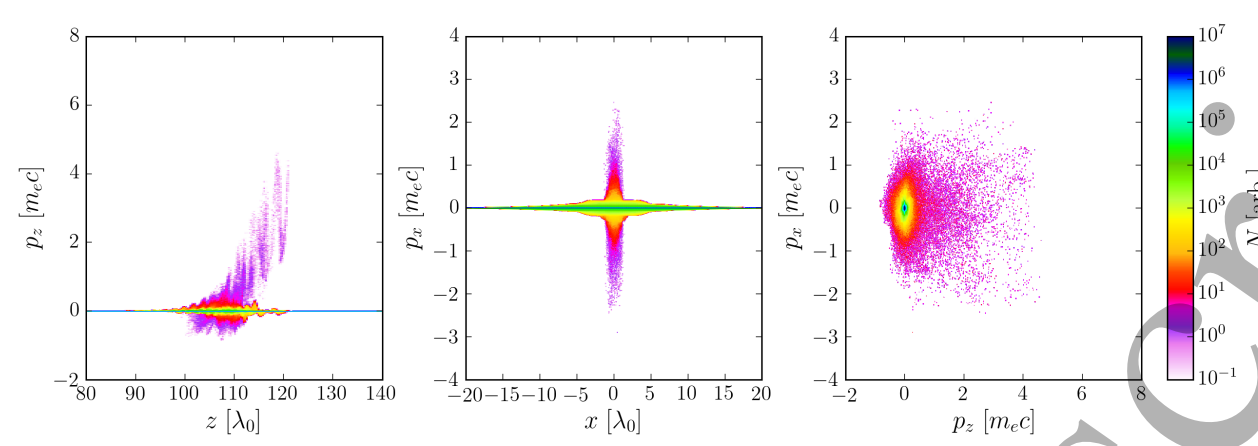

Figure 8: Magnetised simulation electron phasespace distributions at $t=190 \tau_{0}$, following the first collapse. (a) The longitudinal momentum $p_{z}$ versus the longitudinal axis $z$. (b) The transverse momentum $p_{x}$ versus the $x$ axis. (c) $p_{z}-p_{x}$ distribution.

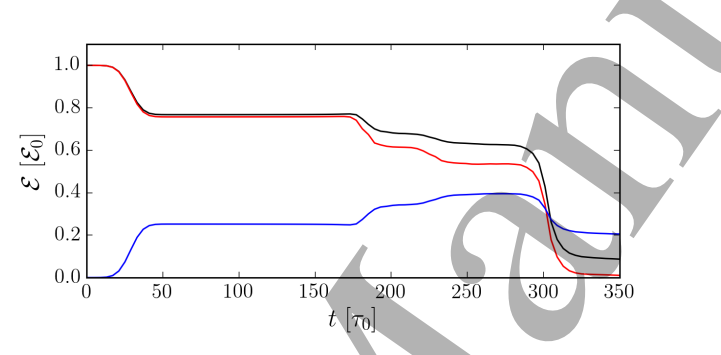

Figure 9: Energy balance over the course of the simulation with $n_{0}=0.15, B=0.5$. The black line shows the total EM energy contained in the simulation $\left(E^{2}+B^{2}\right)$, the red line shows the total radiative energy calculated from the Poynting vector $(|\boldsymbol{E} \times \boldsymbol{B}|)$, and the blue line shows the total kinetic energy of the electrons (ion kinetic energy is negligible). The sharp drop in EM energy at $t \approx 300 \tau_{0}$ corresponds to the laser pulse leaving the simulation box.

soliton formation, localised at the points of collapse. These are distunguished from those produced in the unmagnetised case by the much greater lifespan of the contained solitons. They can be seen as vertical tracks, stationary in space, in figure $7(\mathrm{~b})$ and persist many times longer. Research on post-soliton formation in magnetised plasma is less robust than for unmagnetised plasma, [36, 37, 38], with studies of 3D magnetised post-solitons doubly so. The mechanism of their comparative longevity and other specific details of magnetised post-solitons are beyond the scope of this work, however the topic certainly warrants futher study.

The frequency spectrum is also quite comparable with figure $7(\mathrm{~d})$, showing strong frequency downshift and spectral broadening due to scattering close to collapse and trapping of laser fields inside the cavities.

The production of energetic electrons in this case is similar to the unmagnetised case, which is found only when pulse collapses occur. However from figure 8(b) we observe that the electrons are constrained on the central axis by the magnetic field, effectively collimating the particles in the forward direction. The electron density at the end of the simulation is again comparable, with some electron accumulation on the axis, due to the magnetic field constraining electrons. Again we see no energetic 

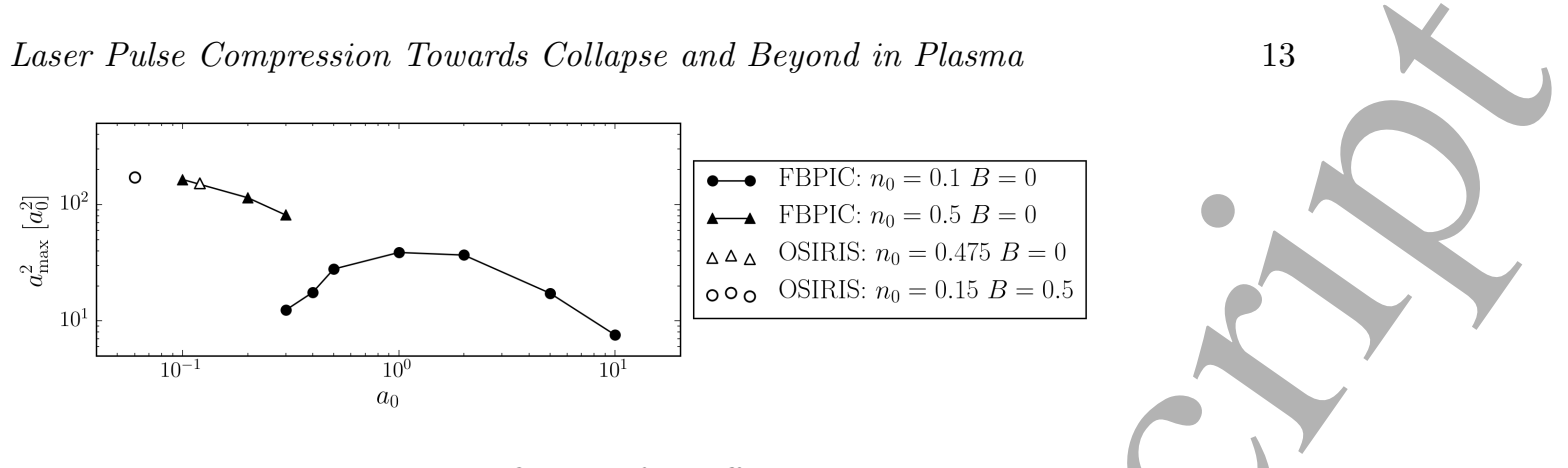

Figure 10: Peak intensity amplification for different initial amplitudes and plasma conditions. The legend gives the code and plasma conditions which produced each result. The OSIRIS results correspond to those discussed in section 3.1 and 3.2. In all cases, when the maximum intensity for the simulation is reached, the pulse is close to lambda-cubic proportions.

ion production, and the final ion density distribution is near-identical to that of the electrons.

The dependence on the magnetic field is very strong, as evidenced by the fact that the rate of compression is comparable to the unmagnetised ease at half the laser amplitude, and less than half the plasma density. We point out once more that a laser pulse and plasma of equivalent parameters without such a strong magnetic field, would not exhibit any self-focusing or self-compression. The real magnetic field strength required to elicit these effects varies with laser wavelength. For example, the value of $\omega_{c} / \omega_{0}=0.5$ for $\lambda_{0} \approx 1 \mu \mathrm{m}$ corresponds to around 5,000 $\mathrm{T}$, which remains a challenge to produce experimentally [39]. However, if we consider infrared sources, such as $\mathrm{CO}_{2}$ lasers with $\lambda_{0} \approx 10 \mu \mathrm{m}$, this is reduced to $500 \mathrm{~T}$, which is more readily available $[40,41]$. Thus this magnetised plasma approach suggests a novel method of compression for long-wavelength or low-amplitude lasers, which are typically more difficult to manipulate $[42,43]$.

\subsection{The case for high amplitudes}

It is also worthwhile to look at the physics as laser amplitude is raised beyond the weakly-relativistic limit. We choose to examine densities of $n_{0}=0.1$ and $n_{0}=0.5$, represented by the circle and cross on figure 3 , respectively. By maintaining $R_{0}=10 \lambda_{0}$, from (6) this requires $D_{0}^{\mathrm{vac}}=3.51$ and 14.14 , respectively. We use FBPIC to simulate several different amplitude cases and present the results in figure 10. As we are interested only in the envelope dynamics up to the first collapse, we leave ions immobile, as the simulations do not extend to the ion motion timescale. Correspondingly we increase the electron particle count to 100 particles per cell distributed as $2 \times 2 \times 25$ in $(r, z, \theta)$ and utilise 5 azimuthal modes.

In overview, we may examine the outcome of a compression by the maximum intensity amplification. This is performed for each simulation at the point of collapse and the results are summarised in figure 10. We see a general downward trend as amplitude is increased, with low amplitude pulses in near-critical density plasma capable of significant amplification, over 150 times the initial intensity, as exhibited by both of the OSIRIS simulations, and the FBPIC simulation with $n_{0}=0.5, a_{0}=0.1$, seen as the collection of points at the far left on figure 10.

For a more detailed examination, we may compare two specific cases. A laser with 

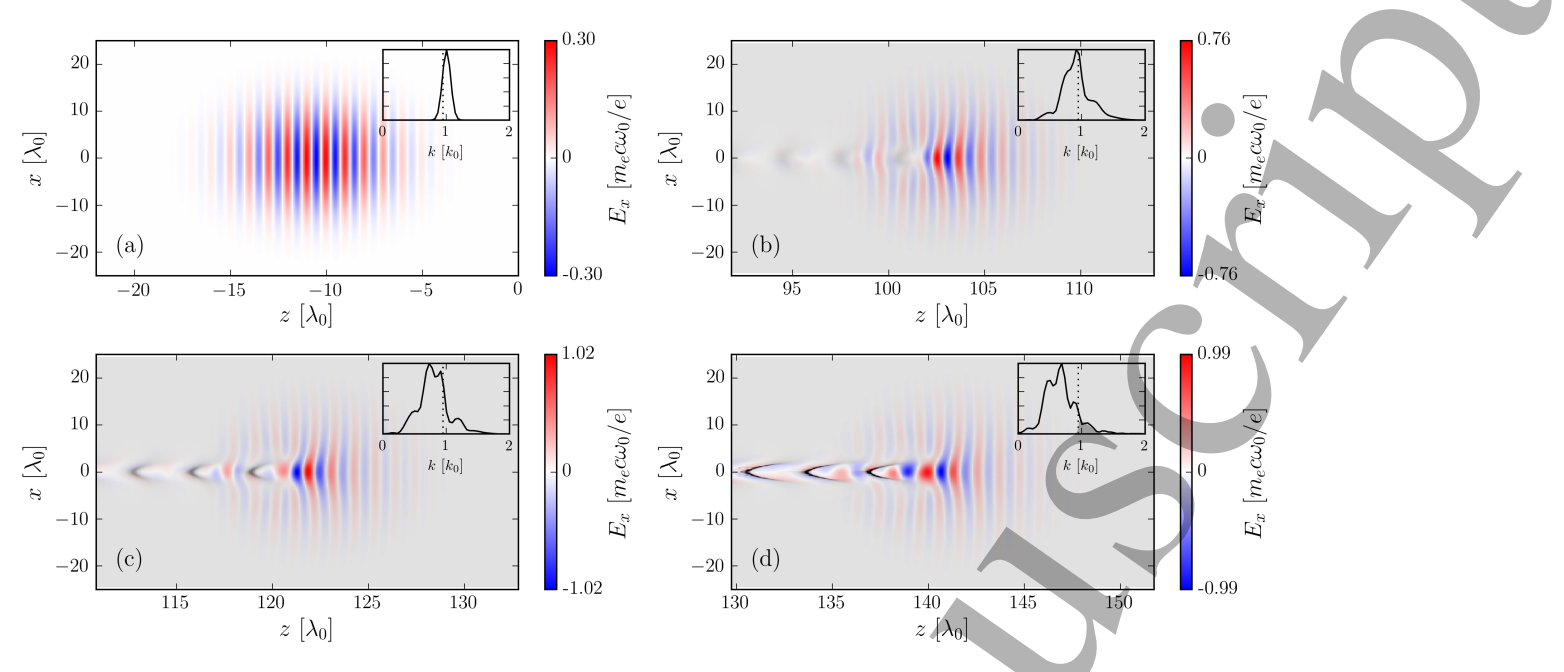

Figure 11: Laser electric field (red/blue) and illustrative plasma density (greyscale) at progressive times, for the case of $n_{0}=0.1, a_{0}=0.3$. The inset plots show the $k$-space spectrum of the $E_{x}$ field along the central axis of each snapshot. The dotted line represents the linear expected value of $k$ based on the plasma refractive index. (a) $t=0$, the initial pulse profile. (b) $t=120 \tau_{0}$, the pulse after entering the plasma. (c) $t=140 \tau_{0}$, the pulse at its minimum extent. (d) $t=160 \tau_{0}$, the pulse post-collapse.

amplitude $a_{0}=0.3$ was simulated for both low, and high density plasma. Both pulses have the same peak amplitude, but due to their differing dimensions, contain different amounts of energy. The pulse in high density plasma contains approximately 4.5 times the energy of the pulse in low density. As such, we expect that the amplifcation factor in high density plasma will be greater than that of the low density plasma, if we are taking the pulses to be spherically symmetric in their respective plasmas. For the long and short pulses we consider the maximum amplification factor calculated from $a / a_{0}=\sqrt{R_{0}^{2} D_{0}}$ comes out to 37 and 19 times the initial amplitude respectively. In fact, the simulation results show amplification factors of 8.6 for the long pulse and 3.4 for the short pulse. This disparity is in line with that discussed in section 3.1.

Some snapshots of the simulation results are shown in figures 11 and 12, showing the laser $E_{x}$ field, electron density and wavenumber spectra at various times for the $n_{0}=0.1$ and $n_{0}=0.5$ simulations respectively. The low-density case in figure 11 shows the initially Gaussian pulse in figure 11(a). In figure 11(b), after propagating some distance into the plasma, the pulse envelope has contracted both longitudinally and transversely as expected, but it is also modulated by the forming plasma wake. The next snapshot figure 11(c) shows the pulse a close-to its minimum spatial extent. We see that although compression is quite symmetric, it is not as strong as it could be, limting to around $2 \lambda_{0}$ in spot-size, and similar longitudinal extent. As propagation continues from figure 11 (c) to $11(\mathrm{~d})$, we see that the frequency downshift continues, and the pulse begins to distort due to the continual downshifting, elongating and trailing in the wake, with frequency components becoming separated [44]. This results in only modest amplification of 10-20 times the initial intensity. In this density regime, spherical compression requires both shorter pulses and higher initial amplitudes than the high-density or magnetised cases. This means lower total energy overall, and 
Laser Pulse Compression Towards Collapse and Beyond in Plasma
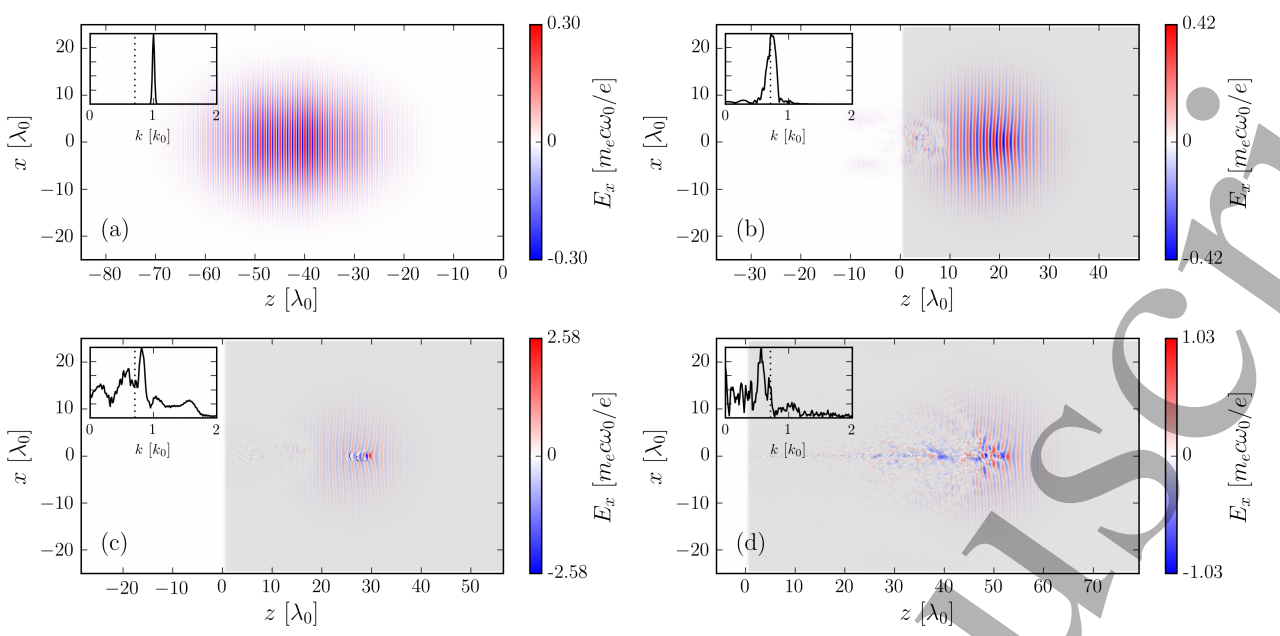

Figure 12: Laser electric field (red/blue) and illustrative plasma density (greyscale) at progressive times, for the case of $n_{0}=0.5, a_{0}=0.3$. The inset plots show the $k$-space spectrum of the $E_{x}$ field along the central axis of each snapshot. The dotted line represents the linear expected value of $k$ based on the plasma refractive index. (a) $t=0$, the initial pulse profile. (b) $t=68 \tau_{0}$, the pulse after entering the plasma (c) $t=80 \tau_{0}$, the pulse at its minimum extent (d) $t=112 \tau_{0}$, the pulse post-collapse.

renders the compression process open to competition from ponderomotive effects. Compounding this, at close-to-threshold laser powers such as these, the propagation distance before collapse is quite long, giving more time for even low growth-rate instabilities to become significant such as pulse modulation due to scattering processes and pulse spectral changes.

Correspondingly, the high density case is shown in figure 12. Again, (a) shows the initial pulse profile, and (b) the pulse after entering the plasma, some reflection is seen as the pulse enters the plasma. Due to the higher plasma density, the time and distance scales for collapse are much shorter than in figure 11. After only $30 \lambda_{0}$ the pulse has reached the lamda-cubic regime, with a fitted spot-size of only $0.7 \lambda_{0}$, and an equivalent duration. This naturally induces strong spectral broadening as seen in the inset of (c), however due to the short propagation distance and high plasma density, no appreciable wake has formed to distort the pulse, we attribute the very compact compressed profile to this. Post-collapse, the pulse disintegrates rapidly, with the remnants continuing to propagate as seen in other simulations.

As the amplitude is raised to $a_{0} \sim 1$, the high density case sees rapid filamentation and disintegration of the pulse, entirely dominating any other envelope processes and making such conditions unsuitable for compression. The low density plasma however exhibits a local amplification maximum. The pulses with moderate amplitudes $a_{0}=1$ and $a_{0}=2$ show amplfication of around 40 times the initial intensity. We offer as explanation that in this regime the compression is fast enough that the wake does not modulate the main pulse much, and distortions from ponderomotive effects are also at a minimum, resulting in relatively clean compression.

As amplitude is further raised, the amplification drops once more, falling monotonically as ponderomotive losses and other destructive processes dominate the

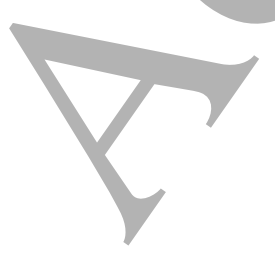


propagation. At $a_{0}=10$, the maximum amplification has fallen to less than one order of magnitude. Spherical compression appears to still be possible in this regime, however, due to ponderomotive focusing and leading-edge etching, it is least described by our model.

In all cases, the compressed profile approaches the lambda-cubic regime, with the 'best' results reaching slightly sub-wavelength dimensions, albeit briefly. In general the higher the amplification, the more closely the compressed pulse approaches the lambda-cubic regime.

\section{Conclusions}

In conclusion, we have demonstrated the viability of spherical compression and subsequent pulse collapse for short-pulse lasers over a wide parameter range. 3D numerical simulation suggests the laser pulses can be compressed to the lambda-cubic regime before they reach collapse. Spherical collapse is accompanied by the significant spectrum broadening, the formation of density cavities and the production of energetic electrons in the forward direction. Multiple pulse collapses can be found during the laser pulse propagation until the pulse energy is sufficiently dissipated to plasma. Such phenomena can occur even for short laser pulses with input energy as small as a few millijoules and thus can be demonstrated experimentally on various laser systems. The inclusion of a magnetic field is/shown to enhance the compression rates at lower densities and amplitudes, and could potentially be used to manipulate longwavelength lasers which are typically difficult to compress below the picosecond scale. While compression can achieve impressive factors of ámplification, energy balance analysis shows that only a small fraction of the total EM energy is contained in the core of the pulse, as such the use of a plasma channel may be beneficial to corral the laser energy towards the axis and improve overall efficiency. While we have exclusively considered one specific case, the coupled nature of the evolution equations derived here afford them the potential to govern the shaping of light into nearly any profile, this offers an exciting outlook for future research.

\section{Acknowledgments}

The authors acknowledge the OSIRIS consortium, a Leverhulme Trust Research Project Grant, the U.K. EPSRC (Grant No. EP/N028694/1 and EP/R006202/1), EuPRAXIA (Grant No. 653782), and National Science Foundation of China (Grant No. 11775144 and 11721091). Numerical simulations have been performed on ARCHER via Plasma HEC Consortium supported by EPSRC (No. EP/L000237/1).

\section{References}

[1] Y. Silberberg. Collapse of optical pulses. Opt. Lett., 15:1282, 1990.

[2] G. Cerullo, A. Dienes, and V. Magni. Spacectime coupling and collapse threshold for femtosecond pulses in dispersive nonlinear media. Opt. Lett., 21:65, 1996.

[3] A. L. Gaeta. Catastrophic collapse of ultrashort pulses. Phys. Rev. Lett., 84:3582, 2000.

[4] A. B. Borisov, A. V. Borovskiy, O. B. Shiyaev, V. V. Korobkin, A. M Prokhorov, J. C. Solem, T. S. Luk, K. Boyer, and C. K. Rhodes. Relativistic and charge-displacement self-channeling of intense ultrashort laser pulses in plasmas. Physical Review A, 45:5830, 1992.

[5] A. Pukhov and J. Meyer-ter Vehn. Relativistic magnetic self-channeling of light in near-critical plasma: Three-dimensional particle-in-cell simulation. Phys. Rev. Lett., 76:3975, 1996. 
[6] C. Ren et al. Compressing and focusing a short laser pulse by a thin plasma lens. Phys. Rev. E, 63:026411, 2001.

[7] O. Shorokhov et al. Self-compression of laser pulses in plasma. Phys. Rev. Lett., 91:265002, 2003.

[8] H. Y. Wang, C. Lin, Z. M. Sheng, et al. Laser shaping of a relativistic intense, short gaussian pulse by a plasma lens. Phys. Rev. Lett., 107:265002, 2011.

[9] F. Sylla, A. Flacco, S. Kahaly, M. Veltcheva, A. Lifshitz, Makla V., E. D'Humières, I. Andriyash, and V. Tikhonchuk. Short intense laser pulse collapse in near-critical plasma. Phys. Rev. Lett., 110:085001, 2013.

[10] H. Leblond, D. Kremer, and D. Mihalace. Collapse of ultrashort spatiotemporal pulses described by the cubic generalized kadomtsev-petviashvili equation. Physical Review A, 81:033824, 2010.

[11] V. Shumakova, P. Malevich, S. Ališauskas, A. Voronin, A. M. Zheltikov, D. Faccio, D. Kartashov, A. Baltuška, and A. Pugžlys. Multi-millijoule few-cycle mid-infrared pulses through nonlinear self-compression in bulk. Nature Communications, 7:12877, 2016.

[12] A. A. Balakin, A. G. Litvak, V. A. Mironov, and S. A. Skobelev. Self-compression of soliton-like laser pulses in the process of self-focusing. Journal of Optics, 19:095503, 2017.

[13] G. Fibich. The Nonlinear Schrodinger Equation-Singular Solutions and Optical Collapse. Springer, 2015.

[14] D. Anderson and M. Lisak. Nonlinear asymmetric self-phase modulation and self-steepening of pulses in long optical waveguides. Phys. Rev. A, 27:1393, 1983.

[15] Z.-M. Sheng, Ma J.-X., Z.-Z. Xu, and W. Yu. Effect of an electron plasma wave on the propagation of an ultrashort laser pulse. J. Opt. Soc. Am. B, 10:122, 1993.

[16] D. Anderson. Variational approach to nonlinear pulse propagation in optical fibers. Physical Review A, 27:3135, 1983.

[17] E. Esarey, P. Sprangle, J. Krall, and A. Ting. Self-focusing and guiding of short laser pulses in ionizing gases and plasmas. IEEE Journal of Quantum Electronics, 33:1879, 1997.

[18] G. Z. Sun, E. Ott, Y. C. Lee, and P. Guzdar. Self-focusing of short intense pulses in plasmas. Physics of Fluids, 30:526, 1987.

[19] T. C. Wilson, F. Y. Li, M. Weikum, and Z. M. Sheng. Influence of strong magnetic fields on laser pulse propagation in underdense plasma. Plasma Phy. Control. Fusion, 59:065002, 2017.

[20] G. Mourou and V. Yanovsky. Relativistic optics: A gateway to attosecond physics. Opt. and Photonic News, 15:40, 2004.

[21] N. M. Naumova, J. A. Nees, I. V. Sokolov, B. Hou, and Mourou G. A. Relativistic generation of isolated attosecond pulses in a $\lambda^{3}$ focal volume. Phys. Rev. Lett., 92:063902, 2004.

[22] G. D. Tsakiris et al. Route to intense single attosecond pulses. New J. Phys., 8:19, 2006.

[23] G. Mourou and Tajima T. More intense, shorter pulses. Science, 331:41, 2011.

[24] A. A. Balakin, A. G. Litvak, V. A. Mironov, and S. A. Skobelev. Self-compression of relativistically strong femtosecond laser pulses during the excitation of a plasma wake wave. EPL, 100:34002, 2012.

[25] A. Pipahl et al. High-intensity few-cycle laser-pulse generation by the plasma-wakefield selfcompression effect. Phys. Rev. E, 87:033104, 2013.

[26] M. Desaix, D. Anderson, and M. Lisak. Variational approach to collapse of optical pulses. J. Opt. Soc. Am. B, 8:2082, 1991.

[27] S. Weng et al. Extreme case of faraday effect: magnetic splitting of ultrashort laser pulses in plasmas. Optica, 4:1086, 2017.

[28] R. A. Fonseca, Silva L. O., F. S. Tsung, V. K. Decyk, W. Lu, C. Ren, W. B. Mori, S. Deng, S. Lee, T. Katsouleas, and J. C. Adam. Osiris, a threedimensional fully relativistic particle in cell code for modeling plasma based accelerators. Lect. Notes Comput. Sci., 2331:342, 2002.

[29] Rémi Lehe, Manuel Kirchen, Igor A. Andriyash, Brendan B. Godfrey, and Jean-Luc Vay. A spectral, quasi-cylindrical and dispersion-free particle-in-cell algorithm. Computer Physics Communications, 203:66-82, 2016.

[30] K. D. Moll and A. L. Gaeta. Self-similar optical wave collapse: Observation of the townes profile. Phys. Rev. Lett., 90:203902, 2003.

[31] B. Zhu, Y.-C. Wu, K.-G. Dong, W. Hong, J. Teng, W.-M. Zhou, L.-F. Cao, and Y.-Q. Gu. Observation of a strong correlation between electromagnetic soliton formation and relativistic self-focusing for ultra-short laser pulses propagating through an under-dense plasma. Physics of Plasmas, 19:102304, 2012.

[32] K. Mima, M. S. Jovanović, Y. Sentoku, Z.-M. Sheng, M. M. Škorić, and T. Sato. Stimulated photon cascade and condensate in a relativistic laser-plasma interaction. Physics of Plasmas, 8:2349, 2001.

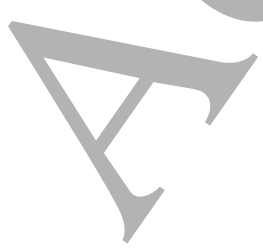


[33] N. M. Naumova et al. Formation of electromagnetic postsolitons in plasmas. Phys. Rev. Lett., 87:185004, 2001.

[34] S. Luan et al. Trapping of electromagnetic radiation in self-generated and preformed cavities. Laser and Particle Beams, 31:589, 2013.

[35] T. Esirkepov, K. Nishihara, S. V. Bulanov, and F. Pegoraro. Three-dimensional relativistic electromagnetic subcycle solitons. Phys. Rev. Lett., 89:275002-1, 2002.

[36] D. Farina, M. Lontano, and S. Bulanov. Relativistic solitons in magnetized plasmas. Phys. Rev. E, 62:4146, 2000.

[37] J. Borhanian, I. Kouraskis, and S. Sobhanian. Electromagnetic envelope solitons in magnetized plasma. Phys. Lett. A, 373:3667, 2009.

[38] W. Feng, J. Q. Li, and Y. Koshimoto. Relativistic soliton formation in laser magnetized plasma interactions. J. Phys.: Conf. Ser., 717:012031, 2016.

[39] U. Wanger et al. Laboratory measurements of $0.7 \mathrm{gg}$ magnetic fields generated during highintensity laser interactions with dense plasmas. Physics of Plasmas, 70:026401, 2004.

[40] J. J. Santos et al. Laser-driven platform for generation and characterization of strong quasi-static magnetic fields. New Journal of Physics, 17:083051, 2015.

[41] V. T. Tikhonchuk, M. Bailly-Grandvaux, J. J. Santos, and A. Poyé. Quasistationary magnetic field generation with a laser-driven capacitor-coil assembly. Physical Review E, 96:023202, 2017.

[42] M. N. Polyanskiy, M. Babzien, and I. V. Pogorelsky. Chirped-pulse amplification in a $\mathrm{CO}_{2}$ laser. Optica, 8:675, 2015.

[43] S. Tochitsky, F. Fuiza, and C. Joshi. Prospects and directions of $\mathrm{CO}_{2}$ laser driven accelerators. AIP Conference Proceedings, 1777:020005, 2016.

[44] Z. Nie et al. Relativistic single-cycle tunable infrared pulses generated from a tailored plasma density structure. Nature Photonics, 12:489, 2018. 